\title{
-NOTES-
}

\section{THE INVERSE OF A STIFFNESS MATRIX*}

By K. E. BISSHOPP (Armour Research Foundation)

It is well known that torsional vibration problems of ten require the computation of latent roots of matrices. Now the usual methods ${ }^{1}$ give these roots in descending order of magnitude while in torsional vibration problems we require the smallest root of the stiffness matrix and then, perhaps, some of the remaining roots in ascending order of magnitude. It is therefore necessary to find the inverse $u^{-1}$ of the given stiffness matrix; $\boldsymbol{u}^{-1}$ is called the flexibility matrix. If its roots are in descending order $p_{1}, p_{2}, \cdots, p_{k}$ then the required roots of the original stiffness matrix in ascending order are $1 / p_{1}, 1 / p_{2}, \cdots, 1 / p_{k}$.

In general it is very difficult to invert a given matrix. The purpose of this note is to show that a special type of stiffness matrix which occurs frequently can be inverted with a small amount of work. Let us consider, for purposes of illustration, $n+1$ discs with moments of inertia $I_{0}, \cdots, I_{n}$ connected by massless elastic shafts of circular** cross section. Let $c_{i}$ be the coefficient of stiffness of the shaft between the $i$ th and $(i+1)$ th discs. Then

$$
c_{i}=G J_{d} / l_{i},
$$

where $l_{i}$ is the length of the shaft in question and $G J_{s}$ is a numerical factor depending on the material and the polar sectional moment of inertia. The application of Lagrange's equations ${ }^{2}$ to the functions representing the kinetic and potential energies of the system respectively yields a system of linear differential equations. Therefore the second order time derivatives can be replaced by $-p_{i}^{2}$, so that in the absence of damping we obtain the following system of algebraic equations in matrix form,

$$
\left(p^{2} I-\bar{u}\right) \theta=0,
$$

where $\theta$ is the column matrix of the normal mode appropriate to any value of $p^{2}$ for which Eq. (2) is satisfied. Since the system is capable of a rigid body rotation, $p^{2}=0$ is a solution and the degree of system (2) can be reduced by unity with the aid of the substitution

$$
I_{0} \theta_{0}+I_{1} \theta_{1}+\cdots+I_{n} \theta_{n}=0,
$$

* From a paper, The use of matrices and normal coordinates in the solution of torsional vibration problems, read at the spring meeting of the Wisconsin section of the Math. Assoc. of America, at Milwaukee, Wis., May 13, 1944. Manuscript received August 18, 1944.

1 R. A. Frazer, W. J. Duncan, and A. R. Collar, Elementary matrices and some applications to dynamics and differential equations, Cambridge University Press, Cambridge, 1st Ed., 1938, Chapters I-V. functions.

** For other than circular cross sections, suitable factors can be obtained from the appropriate torsion p. 139.

2 S. Timoshenko, Vibration problems in engineering, 1st. Ed., D. Van Nostrand Co., New York, 1928, 
which may be looked upon as the orthogonality condition ${ }^{3}$ for the degenerate frequency $p=0$. The stiffness matrix $u$ for the reduced system in which the zero root is absent becomes

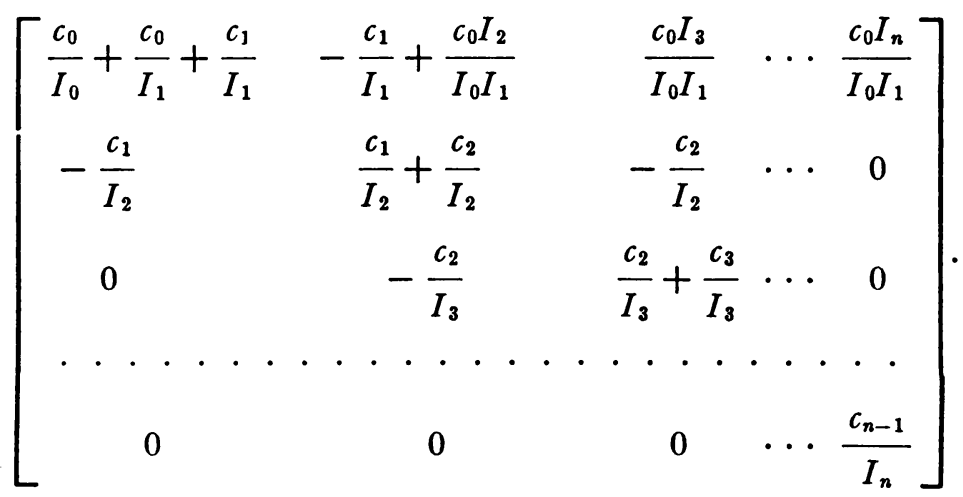

The inverse matrix $u^{-1}=\left(a_{i j}\right)$ may be stated in a convenient form for numerical computation as follows.

If $A_{r}=\sum_{s=r}^{n} I_{s}$, then the $j$ th element in the first row becomes

$$
a_{1 j}=\left[I_{j} /\left(G J \cdot \sum I\right)\right]\left(I_{0} l_{0}-\sum_{r=1}^{j-1} l_{r} A_{r+1}\right) .
$$

When $i \leqq j$ the $i$ th element in the $j$ th column is

and when $i>j$,

$$
a_{i j}=a_{1 j}+\left(I_{j} / G J_{s}\right) \sum_{s=1}^{i-1} l_{s}
$$

$$
a_{i j}=a_{i j}
$$

\begin{tabular}{|c|c|c|c|c|c|}
\hline$n$ & $\begin{array}{l}(1) \\
I_{n}\end{array}$ & $\begin{array}{l}(2) \\
l_{n}\end{array}$ & $\begin{array}{c}(3) \\
I_{n} \times 10^{6} / G J_{\text {s }}\end{array}$ & $\begin{array}{l}(4) \\
A_{n}\end{array}$ & $I_{0} l_{0}-\sum_{1}^{(5)} l_{n}^{n-1} A_{n+1}$ \\
\hline 0 & 181.306 & 30 . & & $25,724.4$ & \\
\hline 1 & $1,328.61$ & 34. & 0.076051 & $25,543.1$ & $5,439.2$ \\
\hline 2 & $1,328.61$ & 34. & 0.076051 & $24,214.5$ & $-817,850$ \\
\hline 3 & $1,328.61$ & 62.2 & 0.076051 & $22,885.9$ & $-1,595,970$. \\
\hline 4 & $21,557.3$ & & 1.23396 & $21,557.3$ & $-2,936,840$ \\
\hline
\end{tabular}

As an application, let us consider the case when $I_{0}=181.306 \mathrm{lb}$.in.sec. ${ }^{2}, I_{1}=I_{2}=I_{3}$ $=1,328.61 \mathrm{lb}$.in.sec. ${ }^{2}, I_{4}=21,557.3 \mathrm{lb}$.in.sec. ${ }^{2}, l_{0}=30$ in., $l_{1}=l_{3}=34$ in., $l_{3}=62.2$ in.,

TABLE I

$G J_{s}=17,470 \times 10^{6} \mathrm{lb}$. in. ${ }^{2}$ All necessary computations are contained in Table I. From the second line of this table $(n=1)$, we obtain immediately

$$
10^{6} a_{11}=I_{0} l_{0} I_{1} / G J_{s} A_{0}=[\mathrm{col} \text {. (5) } \times \text { col. (3) }] / A_{0} .
$$

${ }^{3}$ T. von Kármán and M. A. Biot, Mathematical methods in engineering, McGraw-Hill Book Co., New York, 1940, chapter V, pp. 162-215. 
Equation (4) shows that the remainder of the elements of the first row of $u^{-1}$ can be calculated in order from each succeeding line of the table by performing the operations indicated on columns (3) and (5) respectively. The other elements in the upper righthand corner of $u^{-1}$ then are computed from Eq. (5) which gives for instance when $i=3$ and $j=4$,

$$
\begin{aligned}
10^{6} a_{34} & =10^{6} a_{14}+I_{4} \times 10^{6} / G J_{s} \sum_{n=1}^{n-2} l_{n}=-140.875+1.23396 \times(34+34) \\
& =-56.966 .
\end{aligned}
$$

The inverse matrix now can be completed quickly by filling in the lower left-hand corner according to Eq. (6), so that

$$
u^{-1}=10^{-6} \times\left[\begin{array}{rrrr}
0.0160803 & -2.4179 & -4.7183 & -140.875 \\
0.0160803 & 0.1678 & -2.1326 & -98.920 \\
0.0160803 & 0.1678 & 0.4532 & -56.966 \\
0.0160803 & 0.1678 & 0.4532 & 19.786
\end{array}\right] \text {. }
$$

\section{ON THE PROBLEM OF HEAT CONDUCTION IN A SEMI-INFINITE RADIATING WIRE*}

\section{By ARNOLD N. LOWAN (Math. Tables Project, Nat. Bureau of Standards)}

R. V. Churchill derives the solution of the problem of heat conduction in a semiinfinite radiating wire when the initial temperature is zero, and the boundary temperature is a constant. It is the object of this paper to derive the general solution corresponding to an arbitrary initial temperature distribution when the boundary temperature is a prescribed function of time.

Let $k, c, \rho, s, A, h$ and $\alpha=k / \rho c$ denote the thermal conductivity, specific heat, density, perimeter, cross-sectional area, coefficient of heat transfer, and thermal diffusivity of the wire, respectively. Further, let $a=h s / c \rho A$ and $b=a T_{2}$, where $T_{2}$ is the temperature of the medium. If the wire is sufficiently thin so that the temperature may be assumed to be constant over the entire cross section, the problem becomes one-dimensional and the temperature $T(x, t)$ must satisfy the following differential equation, initial and boundary conditions:

$$
\begin{array}{rlrl}
\left(\frac{\partial}{\partial t}-\alpha \frac{\partial^{2}}{\partial x^{2}}+a\right) T(x, t)=b & (x>0, t>0), \\
\lim _{t \rightarrow 0} T(x, t)=f(x), & (2) ; & T(0, t) & =\varphi(t) .
\end{array}
$$

It is easily verified that the expression

$$
T(x, t)=e^{-a t} u(x, t)+v(x, t)
$$

* Received July 17, 1944.

1 R. V. Churchill, Modern operational methods in engineering, McGraw-Hill Book Company, New York, 1944. p. 119. 